\title{
Educación ambiental hoy: Crisis ambiental y de valores sociales. El papel de la
} educación social ${ }^{9}$

Environmental Education today: Environmental and social values crisis. The role of social education

María Helena Romero Espinosa Doctoranda Universidad Pablo de Olavide maryhelenroes@gmail.com

\section{Resumen}

El objetivo de estudio ha sido plasmar la realidad actual de crisis ambiental y de valores a través de un recorrido histórico de la Educación Ambiental hasta nuestros días. En la redacción histórica se trata el desarrollo evolutivo del concepto de Educación Ambiental, explicando el desarrollo de una Educación Ambiental a nivel local para los ciudadanos. El resultado, es la necesidad de un cambio social y de valores actuales, con la aplicación de la Educación Ambiental como herramienta para este cambio.

\section{Palabras clave}

Crisis ambiental, crisis de valores, educación ambiental, cambio social y ciudadanía.

\begin{abstract}
The aim of the study was to show the current situation of environmental crisis of values, through a historical overview of environmental education to this day. In historical writing the evolutionary development of the concept of environmental education, explaining the motivation for the development of environmental education at the local level as well for the citizens concerned. The result is the need for social change and current values, with the implementation of environmental education as a tool for this
\end{abstract}

\footnotetext{
${ }^{9}$ Recibido 27/8/2015 Evaluado 2/9/2015 Aceptado 1/12/2015
} 
change.

\section{Key words}

Environmental crisis, crisis of values, environmental education, social change and citizenship.

\section{La Educación Ambiental hoy}

Vivimos en una sociedad que cambia aceleradamente, que está sometida a profundas transformaciones y necesita estar continuamente en proceso de actualización interna. Debemos desarrollar nuevas habilidades, poniendo en juego nuevas estrategias que nos ayuden a una mejor integración y a una actuación adecuada en el medio en el que nos desenvolvemos.

Si nos centramos y adentramos en el contexto reglado, el modelo tradicional de educación estructurado en la escuela, generalmente en áreas curriculares, parece no satisfacer todas las necesidades formativas actuales. Estamos ante una necesidad ya señalada por Coombs (1978) en la década de los setenta, y posteriormente por (Faure, 1982) en su estudio encargado por la UNESCO sobre la situación mundial de la educación: "acercar la escuela a la realidad social en la que se encuentra enclavada, poder así satisfacer las necesidades reales del sujeto y cubrir las expectativas sociales" (Faure, 1982, p. 25).

Se habla de una escuela que forme ciudadanos capaces de integrarse y actuar positivamente en su medio, en contraposición al academicismo imperante. Un academicismo centrado en planteamientos ancestrales, al no responder a las necesidades culturales e intelectuales contemporáneas, corre el riesgo de formar ciudadanos con graves carencias educativas, lo cual dificultará su desenvolvimiento en el mundo que les toque vivir, incluso aunque hayan accedido a niveles educativos superiores, tal y como señanalan Reyzabal, M. y Sanz, A. I. (1995). Esta forma de entender la educación (academicismo) se encuentra relacionada con las diferencias entre "enseñar", entendido como transmitir, exponer e instruir y "educar", que se centra en formar y desarrollar íntegramente a la persona de modo que esté preparada para la vida en sociedad. 
Estamos por ello, ante la necesidad de implantar una educación como proceso de cambio intencional, individual, cualitativo y cuantitativo, que una persona ha de realizar en su comportamiento, con finalidades adaptativas y de desarrollo personal, en relación al medio sociocultural en que vive. Es evidente la necesidad de coordinar los aspectos que se trabajan dentro y fuera de la escuela haciéndolos acordes unos con otros.

Los tiempos actuales nos exigen que tomemos el camino de una educación destinada a satisfacer las necesidades formativas y situaciones problemáticas que tendrán que resolver en el futuro los ciudadanos. Muestra de ese cambio son las finalidades educativas escolares postuladas por la UNESCO (1997): Aprender a aprender, Aprender a hacer, Aprender a convivir y Aprender a ser, bien diferente de la aspiración en la alfabetización, que anteriormente expresaba este organismo internacional. Debemos abandonar los planteamientos mecanicistas, dejar de ser meros transmisores de saberes anquilosados y formar a las nuevas generaciones en aquello que realmente necesitan.

Al mismo tiempo hay una urgente necesidad de un cambio de comportamiento en relación a la Naturaleza y al Medio Ambiente, haciéndose patente la necesidad de una adquisición de conocimientos sobre las actuaciones que conducen a los problemas ambientales. Necesitamos saber cómo reparar los daños que ya han sido causados, y cómo evitar que se repitan en el futuro, aspecto que puede ser cubierto por la Educación Ambiental (EA). El tema debe ser estudiado en la escuela y fuera de ella, porque si deseamos cambiar nuestra actitud frente al Medio Ambiente y la Naturaleza, debemos hacer partícipe al sector educativo desde todos sus ámbitos posibles. Tenemos que transmitir la situación en la que nos encontramos desde todas las vías posibles que tengamos de comunicación social.

Desde los años 60, cuando se empezó a cuestionar el modelo de crecimiento establecido y se denunció el impacto que sobre el Medio Ambiente producía, los diagnósticos ambientales han sido muchos. En todos estos informes y manifiestos que van apareciendo a lo largo de los años se plantea la necesidad de adoptar medidas educativas para frenar el creciente deterioro del entorno que nos rodea. La relación entre educación y Medio Ambiente no es nueva, sin embargo, si es cierto, que la EA aporta novedad y 
da propuestas de soluciones, ya que aparece con entidad suficiente como para constituirse en finalidad y objeto de la educación.

A lo largo su existencia, el hombre ha interaccionado con el medio que le rodea y lo ha modificado, por tanto, los problemas ambientales, como venimos apuntando, no son algo nuevo. Sin embargo, lo que hace preocupante la situación actual es la aceleración de esas modificaciones, y la magnitud de sus consecuencias. Los problemas ambientales no aparecen como independientes unos de otros, sino que constituyen elementos que se relacionan entre sí configurando una realidad diferente a la simple acumulación de todos ellos.

De ahí que hoy en día, hablamos de algo más que simples problemas ambientales, nos enfrentamos a una crisis ambiental, para la cual debemos de ir programando vías de solución, incluso ir lanzando programas de prevención. Según Limón (2000, p.30) “La búsqueda de soluciones exige la necesidad de un diseño educacional con proyectos de acción como alternativa de cambio y mejora", para esta búsqueda de soluciones, Limón, nos señala las siguientes cuestiones:

- $\quad$ El análisis de conflictos ambientales reales: que da cuenta de la necesidad de un planteamiento interdisciplinario.

- $\quad$ El grupo de trabajo: que se consolida como una fuerza que se asegura una mayor efectividad en sus conclusiones.

Sin embargo, entendemos que no podemos percibir esta crisis como conflicto en el que determinados argumentos sobre la vida resultan inadecuados. No son estos planteamientos, sino los comportamientos que tenemos ante el ambiente que nos rodea, los recursos con los que contamos y ante las modalidades de consumo y producción masiva.

Todo esto nos lleva a plantearnos la necesidad inmediata de "pautas de acciónprevención" que desde lo local permita acercarnos a la ciudadanía, que es la que debe de ir asimilando dichas "pautas de acción-prevención". Para Limón (2000, p.7) es en el proceso de la educación, desde donde "[...] se ha observado la capacidad que ha tenido 
la persona humana para ir enseñando, a las generaciones futuras, alternativas para asumir su historia y proyectarla en el futuro". Esto nos lleva a unir procesos para desarrollar un cambio de actitudes, quedándose reflejado en la educación como proceso para la puesta en práctica de pautas de acción-prevención marcadas.

Por lo tanto, podemos acordar que es a partir de la educación y los distintos procesos educativos, el que consigamos llevar a cabo, una vía de solución posible a la actual crisis ambiental por la que estamos pasando.

\section{Educación Ambiental y Desarrollo Sostenible}

Una de las primeras obras en las que se podía vislumbrar, lo que años más tarde se conocería con el nombre de Desarrollo Sostenible, es la obra primigenia de Malthus (1766-1834), considerado uno de los padres de la economía moderna y conocido por sus estudios y predicciones demográficas en relación con la producción de alimentos. Este pensador, insistió en la necesidad de valorar económicamente la naturaleza, ya que consideraba que el ambiente posee una capacidad de producción propia, es decir que se trataba de una fuente generadora de riquezas, y que por tanto manifestaba entidad económica. De todas formas, Malthus, no mostró un mayor interés por la cuestión económico- ambiental, centrándose en cuestiones de previsiones demográficas que estudiaba en función al crecimiento del alimento.

Otro pensador más o menos coetáneo al anterior, fue J. Stuart Mill, que destaca por ser un precedente de la sostenibilidad en su libro Principios de economía política. En el libro se hace patente la necesidad de proteger a la naturaleza del crecimiento desenfrenado, puesto que la considera, uno de los elementos más importantes para preservar el bienestar humano, de forma que si se efectúa un uso irracional de la misma, se puede llegar a rendimientos económicos decrecientes.

Se puede evidenciar por tanto, que es desde el siglo XIX, cuando se viene reflexionando sobre la importancia de los recursos de la naturaleza y sus relaciones con el crecimiento económico. Ya en el siglo XIX, ésta marcada etapa economicista irá dando paso a autores como David Ricardo (en Brynjolfsson y McAfee, 2013, p. 50), quien pensaba 
firmemente que los avances tecnológicos desacelerarían y retrasarían el momento en que se produjese el desequilibrio entre naturaleza y presión económica. Esto hoy en día se puede poner en tela de juicio, puesto que no siempre a un desarrollo o avance tecnológico, le sigue o ha seguido a lo largo de la historia, buenas consecuencias ambientales, ya sea desde una dimensión económica o social.

Se podrían mencionar casos muy claros a los que aún hoy no se les encuentra vía de solución, y desde las primeras manifestaciones de atención a la naturaleza en relación con lo económico o social, ha pasado como estamos comprobando dos siglos. Tal puede ser el caso de la pobreza, como factor importante a abordar desde el establecimiento de un Desarrollo Sostenible actual, en el que más tarde entraremos.

Después de estos antecedentes, parece que la historia que rodea al término Desarrollo Sostenible se nos hace más cercana. Ya en el siglo XX, el Informe del Club de Roma en 1972, marcó una pauta en la que por primera vez se establecieron las posibles consecuencias ambientales asociadas al crecimiento de las poblaciones y de sus estilos de desarrollo.

El informe mencionado, abrió una puerta para abordar el problema y en el mismo año se celebró la Conferencia de Estocolmo, tras las primeras voces de alarma, acerca del grado de degradación ambiental en el que el mundo estaba sumergido. Allá por la década de los setenta, la ONU convocó la conferencia en Estocolmo, cuyo principal objetivo era analizar la situación ambiental del planeta y la crisis económica por la que se estaba pasando. Una de las grandes aportaciones de dicha conferencia tiene que ver con las resoluciones y recomendaciones finales, ya que de ellas se desprendieron la necesidad de desarrollar, a escala mundial, programas de EA.

Este fue un reto asumido por la ONU, que en 1974 crea el Programa de Naciones Unidas de Educación Ambiental, aplicado por la UNESCO en 1975. Todo este tipo de acontecimientos enmarcan el nacimiento del Desarrollo Sostenible de las preocupaciones surgidas a comienzos de los setenta, con respecto a la situación mencionada del planeta, y como consecuencia la necesidad de establecer una nueva relación entre el hombre y el medio en el que vive. 
En 1972 con la obra Los límites del crecimiento, se hace referencia a la necesidad de sustentar la naturaleza. Más tarde en 1974, en la Conferencia de Cocoyoc (México) se utilizó por primera vez el concepto de Desarrollo Sostenible que se discutiría en la convención de la UNESCO, celebrada en Quito en 1979. Como resultado es este debate, se llegó al consenso de sustituir tal término de Desarrollo Sostenible por "Nuevo Desarrollo" o tipo de desarrollo, cuyas características más definitorias serían la de "ser integral, global y endógeno”. Esta definición no tuvo aceptación, por lo que siguió prevaleciendo el término primero de Desarrollo Sostenible, puesto de manifiesto en el Informe de la Unión Internacional para la Conservación de la Naturaleza (UINC), publicado en 1980 con el título Estrategia Mundial para la conservación.

Es a partir de la concienciación de tal problema, cuando NN.UU. (Naciones Unidas) encarga el estudio de los conflictos ambientales de nuestro planeta y la tarea le fue encomendada a la Comisión Brundtland. El informe que elabora dicha Comisión, salió a la luz en 1987 después de varios años de trabajo. Fue en 1988, cuando la Comisión del Medio Ambiente, estudiando el concepto, da una nueva definición del término Desarrollo Sostenible, al que denominaron como "aquel que satisface las necesidades de las generaciones presentes sin comprometer las de las generaciones futuras" (1988, p. 67).

De esta definición se desprenden dos ideas que afectan de lleno a la sociedad, que son la idea de necesidades, tanto a nivel de sostenimiento de la naturaleza, como la repercusión que ésta tiene en el desarrollo de las personas. Esta segunda dimensión humana, se resalta en el Informe Brundtland (Naciones Unidas en 1987, p.60) donde expone "satisfacer las necesidades esenciales de los más pobres", como tema importante a abordar desde la EA. Insiste en la generalidad del problema, ya que es un mal que afecta a un sector importante de la sociedad, sin distinción entre países desarrollados y países en desarrollo.

Queremos decir con esto, que aún en los denominados países ricos encontramos grupos desfavorecidos y que no tienen cubiertos los niveles básicos de calidad de vida, a los que habría que atender. Otra de las ideas que se desprende de dicho Informe es el los 
límites. Con este término, se hace referencia a las limitaciones impuestas por la capacidad de carga del ecosistema. Con esto se plantea los límites que existen o deberían existir, en la utilización de los recursos que nos ofrece el planeta.

Con este recorrido por la evolución del término Desarrollo Sostenible, se puede evidenciar un paso de denominación del mismo desde aspectos más económicos en sus comienzos, a aspectos más humanistas con la definición de la Comisión Brundtland. Con esto se puede comprobar, que no sólo importa la dimensión económica, sino que también juegan un papel importante dimensiones como la social y dentro de la misma merecen mayor atención temas como la pobreza en el mundo por ejemplo, que más adelante trataremos.

\section{Educación Ambiental y Desarrollo Sostenible. Evolución histórica.}

Para hacer un recorrido por la evolución del movimiento de EA, vamos a realizarlo por etapas cronológicas, que marcan el desarrollo de dicho movimiento en relación con el Desarrollo Sostenible. 1960-70, marca la etapa de sus principios y es la fundación del Council for Environmental Education de la Universidad de Reading, Inglaterra (1968), que se toma como punto de referencia. Se trata de un periodo en el que el campo que ocupaba la EA se tenía que ir configurando, puesto que conceptualmente estaba aún por determinar. En esta década también hubo que superar dos grandes lagunas, que han marcado lo que se conoce hoy como EA.

En primer lugar uno de los grandes retos era el de ampliar el concepto de Medio Ambiente. Siempre se ha tenido la idea preconcebida de la relación existente entre Medio Ambiente y medio natural, cosa que por otro lado no está mal, pero la EA no sólo abarca a lo ecológico sino que también debe atender dimensiones como la económica, social, política... que inciden directamente en los sistemas ambientales.

Otro gran reto, fue en el ámbito Formal/Reglado, es decir en lo escolar, hacer ver que la EA tenía que ser una dimensión que impregnara todo el currículo. Se refería a que no se entendiera este movimiento educativo, como si de una asignatura "normal", sino que se tratase de instaurar como un "eje transversal" y que impregnase el cuerpo curricular 
entero, trabajándose desde el resto de las materias escolares. En esta ocasión, España se sumó a la iniciativa que se hizo efectiva con la promulgación en 1990 de la LOGSE (Ley Orgánica de Ordenación del Sistema Educativo). Una de sus novedades de esta Ley era precisamente la de incorporación de los denominados "temas transversales", uno de los cuales fue la Educación Ambiental.

En el ámbito No Formal/No Reglado, la década de los 70 está marcada por el despegue de grupos ecologistas e incluso desde países anglosajones se les va dotando de cierta dimensión educativa. También se desarrollaron actividades extraescolares que van creando estructuras de apoyo a la escuela en su acción a favor del Medio Ambiente. Se trata por tanto, de un gran empuje en la evolución y desarrollo del movimiento educativo ambiental.

En la década de los 80 a los 90, se recuerda un importante aumento de experiencias de EA en el ámbito No Formal, como es el caso de los equipamientos ambientales tales como las Granjas-Escuelas, Aulas de la Naturaleza, programas llevados a cabo desde los Ayuntamientos... Hoy por hoy conforman un abanico de posibilidades educativas y lúdicas para los escolares y no escolares, siendo una importante aportación educativa y formativa. Es un periodo en el que el conjunto social (la sociedad al completo) va dándose cuenta de que realmente existe una problemática ambiental y que se trata de un fenómeno global.

Otro acontecimiento importante que marca la evolución del movimiento EA, es la celebración en 1987 del Congreso de Moscú, una de las conclusiones más importantes que de él se extrajeron, fue que no es posible definir las finalidades de la EA sin tener en cuenta las realidades económicas, sociales y ecológicas de cada sociedad y los objetivos que ésta se haya fijado para su desarrollo. De esto se desprende la importancia que tiene la EA Formal, No Formal e Informal en su conjunto, para la construcción de un sistema en el que todos los ámbitos se complementen y apoyen. Se resalta también la necesidad de unir las finalidades de la EA y atender a los objetivos que cada sociedad se marca para su desarrollo. Como se puede apreciar en este recorrido, parece que se va haciendo patente la necesidad de involucrar en el ámbito educativo, en este caso el 
ambiental, parámetros de sostenibilidad.

Y la década de los 90, está marcada por acrecentarse más si cabe, la crisis ambiental, tanto en los aspectos ecológicos (deforestación, cambio climático, agotamiento de recursos...) como en los aspectos sociales (aumento de las migraciones, explosión demográfica, hambrunas enormes...). Dentro de este periodo, cabe mencionar la Conferencia Mundial de Río en 1992. Los resultados de dicha reunión, se concretaron en la Declaración de Río, de la que salió un documento de recomendaciones para actuar.

Al mismo tiempo, se celebró en Río de Janeiro el Foro Global, en el que se reflexionó sobre los temas que se estaban trabajando en la Cumbre anteriormente citada. En dicho Foro se firmaron 32 tratados cuyo principal hincapié estaba en favorecer una EA profundamente comprometida con el cambio social y ecológico, y sobre todo de concienciación de las personas ante los conflictos ambientales.

En los últimos 30 años se pueden enumerar una serie de características que han hecho de la EA más que un movimiento un proceso educativo, que ha ido generando múltiples posibilidades en el campo medioambiental. Se pueden citar las siguientes características de cambio:

- $\quad$ Se ha pasado de la preocupación por los recursos a la preocupación por los modelos donde se decide el uso de tales recursos.

- Se ha pasado del concepto de "ciudadano consumidor" al de "ciudadano como partícipe".

- Un planteamiento actual de la EA es el respeto a todas las culturas, la interacción cultural.

- $\quad$ Se ha pasado de una EA centrada en el ámbito formal (escolar), a una EA que se da tanto en el ámbito formal como en el no formal.

- Uno de los logros más importantes de estos años, ha sido el que la EA ya no está atenta a la pobreza, sino que se trata según Novo (1996, pp. 75102) de una "EA formulada desde los esquemas de quienes valoran la pobreza como el primer gran problema ambiental”. 
De estas características, se desprende tanto la evolución como el cambio que ha seguido el desarrollo del movimiento educativo ambiental, desde la concepción de la EA. Unos inicios caracterizados por aspectos conservadores, que se han ido transformando y en los que ya no se trata a la Educación Ambiental como medio natural exclusivamente, sino que además se extiende a otras dimensiones como la social, económica o política que envuelven a la sociedad en general. Es aquí donde encontramos un importante nexo de unión entre Educación Ambiental y Desarrollo Sostenible.

\section{Breve aproximación a la Educación Ambiental y Desarrollo Sostenible}

Colom (2000, p.30) nos señala esta nueva dimensión que afecta a las personas: "El hambre, la desesperación y la marginación a la que está sometida el $80 \%$ de la población mundial son realidades de las que no podemos esperar facilidades para la paz y la convivencia. Si a todo ello añadimos las acciones del hombre en contra de la pervivencia del planeta (conflictos ambientales que conllevan cada vez mayor intensidad catastrófica), es fácil intuir algunas de las causas que pueden protagonizar un futuro no del todo esperanzador".

Ante tales afirmaciones, el Desarrollo Sostenible se nos presenta como un instrumento capaz de dar respuesta a la problemática mencionada. Se trata por tanto de un paradigma prospectivo, que marca sus pautas de acción de cara a un futuro. Desde un punto de vista ecológico y social, dicho término puede llegar desde el sector educativo, a incidir en las necesidades de las poblaciones presentes, atendiéndolas para que en el futuro los conflictos ambientales sean de menor intensidad. También hace referencia a que las generaciones futuras no vean mermadas las necesidades propias. Dicha afirmación viene a resaltar la definición dada por la Comisión Bruntland en 1987, mencionada anteriormente. Así es como el Desarrollo Sostenible pretende ser una respuesta a la irracionalidad humana, que lleva al hombre a atacar al medio en el que vive.

Y es desde la educación desde donde se puede transmitir una nueva visión del mundo en el que todos contribuyamos a la mejora de la calidad de vida. De ahí, el propiciar desde todos los ámbitos educativos (Formal, No Formal e Informal), una EA que basada en 
valores ambientales propicie una transformación social.

\section{Educación Ambiental y Participación Ciudadana: voluntariado ambiental.}

Partimos de la definición de voluntariado, como la participación desinteresada del hombre en tareas sociales, culturales o cívicas, al servicio de un individuo o de la colectividad. Se puede entender por voluntariado ambiental, lo que se expresa desde la Junta de Andalucía, en el proyecto de Voluntariado Ambiental (2006, p, 6.).

El conjunto de iniciativas desarrolladas por personas que, individualmente o a través de grupos sociales, de forma libre, altruista y sin ánimo de lucro, dedican parte de su tiempo libre a la tarea de mejora del medio ambiente y de conservación de los recursos naturales a través de actuaciones directas, sobre el entorno.

Si hablamos de Voluntariado Ambiental, podemos decir que éste es una de las formas más completas de participación social en la defensa del medio. Se entiende con esto, que dicha acción es una manifestación ciudadana de participación en pro de la mejora de las condiciones medioambientales actuales.

Puesto que partimos de la base que la participación ciudadana es el factor más importante a la hora de desarrollar y llevar a cabo actividades de índole ambiental, no cabe duda de que el voluntariado ambiental desempeñe una labor primordial hacia el medio que nos rodea.

Este compromiso solidario y altruista con el medio y la sociedad, según la Junta de Andalucía (2006, p.11) en sus programas de Voluntariado Ambiental:

Facilita la acción directa sobre los problemas del entorno, pero sobre todo es una potente

Estrategia de Educación Ambiental, que con un desarrollo adecuado puede producir un doble efecto: en primer lugar, posibilitando el cambio de las actitudes y comportamientos de los propios voluntarios y, en segundo lugar, influyendo con su acción sobre el resto de la sociedad.

\section{Plan Andaluz de Medio Ambiente (PAMA), subprograma de Voluntariado Ambiental:}

En la actualidad aumenta la preocupación social en materia de Medio Ambiente motivando cada vez más a los ciudadanos, que participan activamente en la resolución de problemas ambientales por medio de la acción del voluntariado. Es por esto, por lo 
que la Consejería de Medio Ambiente promociona el voluntariado ambiental como forma de facilitar la participación de la sociedad andaluza en la prevención o, corrección de los problemas ambientales de su ámbito.

Por todo ello, la Consejería en este intento de promover la participación social, contempla dentro del Plan Andaluz de Medio Ambiente (PAMA) (2006, p.7) el subprograma de voluntariado ambiental, con el objetivo de:

Promover la participación voluntaria de personas que individualmente o a través de grupos sociales, y de forma libre, altruista y sin ánimo de lucro, dediquen parte de su tiempo libre a la tarea de mejora del Medio Ambiente, y conservación de los recursos naturales.

En dicho documento, de obligada referencia para temas de desarrollo, se establecen unos principios básicos de: Solidaridad, Participación, Cooperación, Autonomía, Integración Social. Al mismo tiempo se define qué son los Proyectos locales de voluntariado, que entendemos como actividades promovidas por asociaciones y e instituciones locales (Ayuntamientos y mancomunidades), en ellas los voluntarios desarrollan una importante labor en su entorno cercano. Las tareas a desarrollar en este tipo de proyectos pueden encuadrarse en algunas de las siguientes categorías:

1. Conservación del litoral. Entre las actividades que se desarrollan en estos entornos pueden citarse la realización de tareas de mantenimiento y conservación de ecosistemas litorales, especialmente trabajos en playas, recogida de residuos, limpiezas de fondos marinos e información y educación ambiental, de los que se seleccionaron 9 proyectos lo que constituye el $6 \%$ del total.

2. Medio Ambiente urbano. En esta categoría de acción voluntaria se desarrollan tareas de mejora de la calidad ambiental de la ciudad, restauración de elementos urbanos y acciones en espacios verdes urbanos y periurbanos, con 50 proyectos siendo el $31 \%$ del total.

3. Defensa del medio forestal. Las actividades de defensa del patrimonio forestal andaluz pueden versar sobre actuaciones de vigilancia en épocas de riesgo, 


\section{RESED}

(C) RESED. ISSN: 2341-3255

de sensibilización e información a población rural y visitante, de mantenimiento y conservación y de reforestación participativa, también con 50 proyectos siendo el $31 \%$ del total.

4. Espacios naturales. Acciones de preservación de las características ecológicas singulares de un espacio natural, colaboración en el mantenimiento del mismo y sus recursos (senderos, señalización, equipamientos...) y acogida e información a visitantes, en la que se seleccionaron 32 proyectos el $20 \%$ del total.

5. Conservación de la biodiversidad. Proyectos centrados en la conservación de especies de fauna y flora, mediante la realización de tareas de vigilancia de especies protegidas y amenazadas (aves, mamíferos marinos, reptiles...), colaboración en actividades de investigación, censos... y conservación de sus hábitat, con 19 proyectos significando el $12 \%$ del total.

Tras esta interesante aportación, podemos comprobar, que a nivel local, se cuenta con una red importante de voluntariado que en sus distintas categorías trabajan a favor del medio y por el medio. Proponemos que este tipo de actividades pueden ser promovidas por los Ayuntamientos, que a su vez pueden crear un grupo dinámico de voluntarios que participen y colaboren en el entorno que les rodea, es decir, por su municipio. Con este estudio nos gustaría invitar a los municipios a fomentar y contribuir con el voluntariado ambiental, a fin de obtener una mejora en la calidad de vida de todos.

\section{Conclusiones y debate}

Entendemos que es una buena forma de educar en valores sociales a la ciudadanía, utilizando la participación activa y propiciando cada vez más la implicación de los ciudadanos. Bajo el lema "Por un pueblo mejor, sin ti es imposible" (una propuesta que se lanza desde aquí para diseñar actividades tipo), podemos llegar a los ciudadanos para involucrarlos en tareas de voluntariado ambiental e implicarlos en la conservación del medio en el que se desenvuelven haciéndoles partícipes del cuidado que requiere el legado que dejaremos a las futuras generaciones. 
Tras todo esta revisión de la literatura, en relación con el tema que nos ocupa, podemos llegar a la necesidad de una reflexión importante por parte de los gobernantes y nos gustaría hacer una última aportación sobre el importante papel de la Educación Social, como disciplina que debe abordar esta temática, si bien no en exclusividad, ya que hemos hablado de la importancia de la transversalidad, sí que lo debiera de hacer como impulsora de los cambios y concienciación de los ciudadanos.

Consideramos necesario comenzar a pensar en desarrollar intervenciones en, sobre y para el Medio Ambiente que contemplen como metas, no sólo el bienestar de las generaciones actuales, sino que se prevea la sana subsistencia de las futuras.

Por lo tanto, el modelo de desarrollo socioeducativo que se requiere es un modelo de Desarrollo Sostenible cuyas herramientas sea una educación centrada en el Medio Ambiente. Estas funciones pueden y deben desarrollarse tanto a través de la Educación Social como de la EA. El reto de las dos será, por tanto, el de promover una nueva relación de la sociedad con la naturaleza, a fin de procurar a las generaciones actuales y futuras un desarrollo personal y colectivo más justo, equitativo y sostenible, que pueda garantizar la conservación del soporte físico-biológico sobre el que se sustenta. El desafío es ante todo, conseguir una educación para la acción social, educativa y ambiental.

Han de facilitar, desde una aproximación global e interdisciplinar, la comprensión de las complejas interacciones entre las sociedades y los ecosistemas que las posibilitan. Todo esto a través de un mejor conocimiento de los procesos ecológicos, territoriales, económicos, sociales y culturales, es decir, análisis crítico del problema socio ambiental y su relación con los modelos de gestión y las acciones humanas.

Teniendo en cuenta estas nociones, a lo largo de este artículo y partiendo de la realidad de la problemática ambiental existente, queremos hacer una aclaración de los fines a seguir por ambas materias hasta llegar a lo que consideramos que será la futura línea de investigación a seguir por ambas, es decir, a la consecución de una buena intervención educativa socio ambiental. 
Vemos que la Educación Ambiental, debe ser considerada, ya que lo es, como una de las dimensiones de la educación y más explícitamente de la Educación Social. Al igual que aquella debe buscar que los sujetos consigan su adaptación social que les capacite para la convivencia y participación en la comunidad, por lo que según Amador (2003), debe favorecer y promover las relaciones humanas, a la vez que debe conseguir la madurez social del individuo. Vemos entonces que la Educación Social y la Educación Ambiental aparecen por lo tanto, como intervención socioeducativa y ambiental, como praxis, con finalidades idénticas a niveles teóricos, objetivos, metodología y en muchas ocasiones contenidos compartidos.

Lejos de mantener líneas separadoras, aparecen interacciones e interdisciplinaridad que va a enriquecer y favorecer la comprensión de la crisis ambiental, de carácter social, que es el origen de los desequilibrios ambientales que faciliten la toma de decisiones superadoras de los mismos (Caride y Meira, 2001).

Esta forma, la educación que se refiere a lo ambiental, dentro del contexto de la Educación Social, tiene la ventaja de entenderse, al tiempo que facilita respuestas a las necesidades de sostenibilidad. Todo ello, dentro del bienestar general en un Medio Ambiente equilibrado que viene a facilitar el desarrollo y el progreso de la vida (Rodríguez, 2005).

A su vez, la Educación Ambiental debe ser una disciplina cuya intervención debe tener en cuenta los aspectos educativos y sociales ya que las personas, los grupos y las comunidades son los afectados y beneficiarios de las transformaciones en el Medio Ambiente.

De aquí que estemos en el convencimiento de que la integración de ambas disciplinas como expone (Rodríguez, 2005), tiene la posibilidad de extenderse y dar respuesta a las necesidades de sostenibilidad. Si incorporamos la idea de que el bienestar de los individuos no sólo en calidad de vida restringida al ámbito social, sino que dentro del bienestar está también la posibilidad de disfrutar de un Medio Ambiente equilibrado y armónico, podemos concluir que dentro de las necesidades básicas de los sujetos, también está explicito la sustentabilidad del entorno. 
En este caso, la Educación Social completa a la educación general del individuo, teniendo como objeto el logro de su madurez social, entre otros. Convirtiéndose así en un aspecto importante de la educación. Esta educación incluye la formación cívicodemocrática, que desarrollará valores sociales, orientados al comportamiento democrático y al compromiso de construcción social.

Igualmente nos gustaría resaltar e insistir, que en el concepto de Educación Social, está implícita la doble vertiente de la educación en general: desarrollo individual y desarrollo social de los sujetos. Ya que en la medida que el sujeto se desarrolla, la sociedad también lo hace; según el sujeto se transforma, la sociedad se transforma; en definitiva, en tanto en cuanto el sujeto aprende, "la sociedad aprende". Es en esta línea y a propósito de los aspectos sociales que llevan al desarrollo, donde consideramos que se hace necesario incluir el término de Desarrollo Sostenible: ambiental, económico, político, cultural y por supuesto, cívico de la vida del sujeto en la comunidad.

En definitiva, el fin último a seguir en la intervención social que unifique los aspectos tanto educativos como medioambientales, será el de educar a los sujetos hacia el compromiso y la transformación de la sociedad, hacia un desarrollo sostenible tanto ecológico como social. Esta intervención va a requerir de un aprendizaje, mediante el que se consigan una serie de actitudes investigadoras y metodológicas interdisciplinares, para la consecución del conocimiento de la globalidad social, y devenir el papel de la educación socio ambiental en ella.

\section{Referencias bibliográficas}

Amador, L. V. (2003). Proyecto Docente. (Sin publicar). Sevilla: Universidad Pablo de Olavide.

Benayas, J. (1992). Paisaje y Educación Ambiental: evaluación de cambios de actitudes hacia el entorno. Madrid: Servicio de Publicaciones del MODT.

Brynjolfsson, E. y McAfee, A. (2013). Cómo la revolución digital está accelerando la innovación, aumentando la productividad y transformando irreversiblemente el 
empleo y la economía. Barcelona: Antony Bosch ed. Educación Ambiental: conceptos y propuestas. Editorial CCS

Caride, J. A y Meira, P. A. (2001). Educación Ambiental y desarrollo humano. Barcelona: Ariel

Caride, J. A. (2003). Las identidades de la Educación Social. Cuadernos de Pedagogía, n. 321 , p. $48-51$

Colom, A. (2000). Desarrollo Sostenible y Educación para el desarrollo. Barcelona: Ediciones OCTAEDRO.

Coombs, P. H. (1978). La crisis mundial de la educación. Nuevas perspectivas. Madrid: Santillana.

Faure, E. (1982): Aprender a ser. Madrid: Alianza/UNESCO.

González Gaudiano, E. (2001). Educación Ambiental: historia y conceptos a veinte años de Tbilisi, México, Sitesa. En: Caride, J. A. y Meira, P. Á. (2001). Educación ambiental y desarrollo humano. Barcelona: Ariel

Iglesias L. y Meira P. (2007). De la educación ambiental a la educación social o viceversa. Educación Social: revista intervención socioeducativa, núm. 35

Limón Domínguez, D. (2000). Pedagogía Ambiental: propuesta de cambio para una sociedad comprometida. Barcelon: PPU.

Madariaga, C. y Cobelo, C (2003). La Educación Ambiental como herramienta social. En: I Jornadas Argentino-Chilenas de Educación Ambiental. 13 al 16 de noviembre de 2003 Málaga: Aljibe

Novo Vilaverde, M. (1996). La Educación Ambiental formal y no formal: dos sistemas complementarios. Revista Iberoamericana de Educación, nº 11, mayo/agosto 96, pp. 75- 102 Novo, M. (1995). La Educación Ambiental. Bases éticas, conceptuales y metodológicas. Madrid: Universitas. 
OUR COMMON FUTURE (1987). World Commission on Environment and Development (WCED). Oxford: University Press, p. 43.

Reyzabal, M. y Sanz, A. I. (1995). Los ejes transversales: aprendizajes para la vida. Madrid: Escuela Española.

Reyzabal, M. y Sanz, A. I. (1995). Los ejes transversales: aprendizajes para la vida. Madrid: Escuela Española.

Rodríguez, M. (2005). Introducción a la educación socioambiental desde la pedagogía social.

Rodríguez, M. (2006). Integración de la Educación Social y la Educación Ambiental. Revista Global Hoy [en línea], n. 8. Disponible en: http://cort.as/aC9g

Sáez, J. (1995). La Educación Ambiental ¿una práctica fallida? En: Ortega, J. (comp). Educación Ambiental: cuestiones y propuestas. Murcia: Caja Murcia., p. 159174

Sáez, J. (2007). Pedagogía Social y Educación Social. Historia, Profesión y Competencias. Madrid: Pearson.

Sáez, J. y Campillo, M. (1997). Luces y sombras de la Educación Ambiental. En: Sáez, J. (Coord.) Transformando los contextos sociales: la educación a favor de la democracia. Barcelona; Murcia: DM

Sauvé, L. (2000). Para construir un patrimonio de investigación en Educación Ambiental. Tópicos en Educación Ambiental. n² (5), p. 51-69

Sauvé, L. (2006). Perspectivas curriculares para la formación de formadores en Educación Ambiental. En: Reflexiones sobre educación ambiental: artículos publicados en la Carpeta Informativa del CENEAM 2000-2006. Madrid: Ministerio de Medio Ambiente, p. 219-232. 
Sosa, N. M. (1990). Ética ecológica: necesidad, posibilidad, justificación y debate. Madrid: Libertarias, pp. 49- 50

Stuart Mill, J. (2008). Principios de economía política. Madrid: ed. Síntesis.

Terradas, J. (1971). Ecología hoy. El hombre y su medio. Barcelona: Teide

Terradas, J. (1979). Ecología y educación ambiental. Barcelona. Omega

UNESCO (1997). Una educación para el segundo milenio. París: UNESCO.

VV. AA. (1994). Construyendo el futuro: Foro Internacional de ONG y Movimientos Sociales: Tratados Alternativos de Río 92. [Madrid]: Centro de Publicaciones, Ministerio de Obras Públicas, Transportes y Medio Ambiente

VV.AA. (1993). Declaración de río sobre el medio ambiente y el desarrollo y programa 21: Informe de la Conferencia de las Naciones Unidas sobre el Medio Ambiente y el Desarrollo. Río de Janeiro, 3 al 14 de junio de 1992. Vol. I, Resoluciones aprobadas por la Conferencia de acciones Unidas. New York: Naciones Unidas

VV.AA. (1998). Pedagogía Social: Revista Interuniversitaria, n. 2. (Educación Ambiental, desarrollo y cambio social)

\section{Referencias en Red:}

Congreso de Moscú (1987). Recuperado de: http://cort.as/aCAT

Proyectos Locales de Voluntariado Ambiental. Recuperado de: http://cort.as/aCAu

Voluntariado Ambiental. Recuperado de: http://cort.as/aCAZ 